\title{
Mechanisms of $\mathrm{Ca}^{2+}$ Handling in Zebrafish Ventricular Myocytes
}

\author{
Elisa Bovo, Alexey V. Dvornikov, Stefan R. Mazurek, Pieter P. de Tombe, and Aleksey V. \\ Zima \\ Department of Cell and Molecular Physiology, Loyola University Chicago, Stritch School of \\ Medicine, 2160 South First Avenue, Maywood, Illinois 60153
}

\section{Abstract}

Zebrafish serves as a promising transgenic animal model that can be used to study cardiac $\mathrm{Ca}^{2+}$ regulation. However, mechanisms of sarcoplasmic reticulum (SR) $\mathrm{Ca}^{2+}$ handling in zebrafish heart have not been systematically explored. We found that in zebrafish ventricular myocytes the action potential (AP)-induced $\mathrm{Ca}^{2+}$ transient is mainly $(80 \%)$ mediated by $\mathrm{Ca}^{2+}$ influx via L-type $\mathrm{Ca}^{2+}$ channels (LTCC) and only $20 \%$ by $\mathrm{Ca}^{2+}$ released from the SR. This small contribution of the SR to the $\mathrm{Ca}^{2+}$ transient was not the result of depleted $\mathrm{SR} \mathrm{Ca}^{2+}$ load. We found that the ryanodine receptor (RyR) expression level in zebrafish myocytes was $78 \%$ lower compared to rabbit myocytes. In permeabilized myocytes, increasing cytosolic $\left[\mathrm{Ca}^{2+}\right]$ from 100 to $350 \mathrm{nM}$ did not trigger SR $\mathrm{Ca}^{2+}$ release. However, an application of a low dose of caffeine activated $\mathrm{Ca}^{2+}$ sparks. These results show that the zebrafish cardiac RyR is not sensitive to the mechanism of $\mathrm{Ca}^{2+}$ induced $\mathrm{Ca}^{2+}$ release. Activation of protein kinase A by forskolin increased phosphorylation of the RyR in zebrafish myocardium. In half of the studied cells, an increased $\mathrm{Ca}^{2+}$ transient by forskolin was entirely mediated by augmentation of LTCC current. In the remaining myocytes, the forskolin action was associated with an increase of both LTCC and SR $\mathrm{Ca}^{2+}$ release. These results indicate that the mechanism of excitation-contraction coupling in zebrafish myocytes differs from the mammalian one mainly because of the small contribution of $\mathrm{SR} \mathrm{Ca}^{2+}$ release to the $\mathrm{Ca}^{2+}$ transient. This difference is due to a low sensitivity of RyRs to cytosolic $\left[\mathrm{Ca}^{2+}\right]$.

\section{Keywords}

Zebrafish; Sarcoplasmic reticulum; $\mathrm{Ca}^{2+}$-induced $\mathrm{Ca}^{2+}$ release; Ryanodine Receptor; Phosphorylation; Heart

\section{INTRODUCTION}

Contraction of an adult mammalian cardiomyocyte vitally relies on well-regulated intracellular $\mathrm{Ca}^{2+}$ homeostasis. During an action potential (AP), a small inward $\mathrm{Ca}^{2+}$ current via L-type $\mathrm{Ca}^{2+}$ channels (LTCC) triggers global $\mathrm{Ca}^{2+}$ release from the sarcoplasmic reticulum (SR) by activating ryanodine receptor (RyR; type 2) $\mathrm{Ca}^{2+}$ release channels. This mechanism of cardiac excitation-contraction coupling (ECC) is known as $\mathrm{Ca}^{2+}$-induced $\mathrm{Ca}^{2+}$ release (CICR) [13]. For relaxation to occur, cytosolic $\mathrm{Ca}^{2+}$ has to be pumped back

Corresponding author: Aleksey V. Zima, Department of Cell and Molecular Physiology, Loyola University Chicago, Stritch School of Medicine, 2160 South First Avenue, Maywood, Illinois 60153, Phone: (708) 216-1184, Fax: (708) 216-6304, azima@ lumc.edu. 
into the SR by the $\mathrm{Ca}^{2+}$-ATPase (SERCA) and extruded through the sarcolemma out of the cell mainly by the $\mathrm{Na}^{+}-\mathrm{Ca}^{2+}$ exchanger (NCX). Abnormalities in $\mathrm{Ca}^{2+}$ homeostasis can lead to contractile dysfunctions and arrhythmias under different pathological conditions including heart failure (HF). Thus, insight into the molecular mechanisms that underlie such abnormalities is important for devising effective therapeutic strategies to improve heart function during $\mathrm{HF}$.

In the last few decades, a significant effort has been made in understanding how alterations of RyR function during different pathological conditions can lead to SR $\mathrm{Ca}^{2+}$ mishandling. The cardiac RyR is a giant multimolecular complex composed of many different accessory proteins including several protein kinases and phosphatases [5;15;19]. A proper arrangement of this complex structure is critical for normal $\mathrm{SR} \mathrm{Ca}^{2+}$ regulation. RyR function is also regulated by various post-translational modifications such as phosphorylation and redox modification. These post-translational modifications have potential to impair normal binding of accessory proteins thereby causing RyR dysfunction. For example, it has been suggested that hyper-phosphorylation of the RyR either by protein kinase A (PKA) [20] or $\mathrm{Ca}^{2+}$ / Calmodulin-dependent kinase type II (CaMKII) [1] destabilizes SR $\mathrm{Ca}^{2+}$ homeostasis leading to generation of $\mathrm{Ca}^{2+}$-mediated cardiac arrhythmias in HF. Studying transgenic mouse models with specific RyR mutations relevant to human cardiomyopathies provides particularly important insight into molecular mechanisms that may cause abnormalities in $\mathrm{Ca}^{2+}$ regulation in cardiac diseases [17;28]. However, progress in this field is limited due to high cost and long time required for the generation of a transgenic mouse. Thus, using alternative cost-efficient transgenic animal models with short lifecycle would significantly contribute to the understanding of the molecular mechanisms of RyR regulation during health and diseases.

In recent years, the zebrafish has emerged as a promising experimental model for a variety of genetic studies due to the ease by which transgenic animals can be generated. In contrast to mouse, zebrafish have shorter lifecycles, larger hatch sizes and are easy to maintain. Similar to mammals, the zebrafish ventricular myocyte possesses the major components of ECC $[22 ; 25 ; 30]$. Furthermore, electrophysiological properties of zebrafish cardiac myocyte (e.g. shape of AP) closely resemble human [10], making this animal model suitable for study of ion channel mutations relevant to human pathologies. However, little information is available regarding zebrafish cardiac $\mathrm{SR} \mathrm{Ca}^{2+}$ handling and $\mathrm{RyR}$ regulation. The aim of this study is to characterize mechanisms of SR $\mathrm{Ca}^{2+}$ regulation in ventricular myocytes isolated from zebrafish hearts. We found that the SR of zebrafish ventricular myocytes contains functional RyRs, however, with very low sensitivity to cytosolic $\left[\mathrm{Ca}^{2+}\right]$. RyR activity can be increased with a low dose of caffeine and by RyR phosphorylation. Because RyRs in zebrafish myocytes are almost inactive under basal conditions, zebrafish could be a useful animal model to study human RyR mutations in cellular surroundings that are not contaminated by "noise" from endogenous RyRs. 


\section{MATERIALS AND METHODS}

\section{Myocyte Isolation}

All animal experiments were performed according to protocols approved by the Institutional Animal Care and Use Committee of Loyola University, and complies with US regulations on animal experimentation. Zebrafish (Danio rerio) were anesthetized using tricaine ( 0.4 $\mathrm{mg} / \mathrm{L}$ ) and euthanized by immersion in ice-cold water for $5 \mathrm{~min}$. Single cardiac myocytes were isolated by enzymatic digestion, modified from [10;30]. Briefly, zebrafish hearts were quickly excised and perfused $(0.5 \mathrm{ml} / \mathrm{min})$ for $30 \mathrm{~min}$ at room temperature with a solution containing (in mM): $\mathrm{NaCl} 100, \mathrm{KCl} 10, \mathrm{MgSO}_{4} 4, \mathrm{KH}_{2} \mathrm{PO}_{4} 1.2$, Taurine 50, glucose 20 and HEPES 10 (pH was adjusted to 6.9 with $\mathrm{NaOH}$ ). Perfusion solution was supplemented with bovine serum albumin (BSA; $1 \mathrm{mg} / \mathrm{ml}$ ), collagenase (type 2, Worthington; $0.2 \mathrm{mg} / \mathrm{ml}$ ) and trypsin (type IX-S, Sigma; $0.12 \mathrm{mg} / \mathrm{ml}$ ). $50 \mu \mathrm{M}$ of EGTA and $37.5 \mu \mathrm{M}$ of $\mathrm{Ca}^{2+}$ were also added to perfusion solution. At the end of perfusion, atrium and bulbous were removed and the heart was placed in $1 \mathrm{ml}$ of the low $\mathrm{Ca}^{2+}$ solution with BSA $(1 \mathrm{mg} / \mathrm{ml})$ without enzymes. Next, the tissue was gently torn up into small pieces followed by $30 \mathrm{~min}$ rest at room temperature before adding $\mathrm{Ca}$. Then $\left[\mathrm{Ca}^{2+}\right]$ was increased gradually to $1 \mathrm{mM}$. The cell suspension was filtered through a nylon mesh and stored at room temperature up to 8-10 hours. Rabbit ventricular myocytes were isolated from New Zealand White rabbits (2-2.5 $\mathrm{kg}$ ) according to the procedure described previously [12]. Rabbits were anaesthetized with sodium pentobarbital (50 mg/kg I.V.). Following thoracotomy hearts were quickly excised, mounted on a Langendorff apparatus, and retrogradely perfused with Liberase (Roche Applied Science, Indianapolis, IN) Blendzyme-containing solution at $37^{\circ} \mathrm{C}$. Digested tissue was then minced, filtered, and washed in a MEM solution containing $\mathrm{Ca}^{2+}(50 \mu \mathrm{M})$ and bovine serum albumin $(10 \mathrm{mg} / \mathrm{ml})$. Chemicals and reagents were purchased from SigmaAldrich and Fisher Scientific unless otherwise stated. All experiments were performed at room temperature $\left(20-24^{\circ} \mathrm{C}\right)$.

\section{Confocal microscopy}

Changes in $\left[\mathrm{Ca}^{2+}\right]_{\mathrm{i}}$, were measured using laser scanning confocal microscopy (Radiance 2000 MP, Bio-Rad, UK and LSM 410, Zeiss, Germany) equipped with a $\times 40$ oil-immersion objective lens (N.A=1.3).

Measurements of $\left[\mathrm{Ca}^{2+}\right]_{i}$ in intact myocytes-To record $\left[\mathrm{Ca}^{2+}\right]_{\mathrm{i}}$ we used the high affinity $\mathrm{Ca}^{2+}$ indicator Fluo-4 (Molecular Probes/Invitrogen, Carlsbad, USA). To load the cytosol with the $\mathrm{Ca}^{2+}$ dye, cells were incubated at room temperature with $10 \mu \mathrm{M}$ Fluo-4/AM for 15 minutes followed by a 20 minute wash. Fluo- 4 was excited with the $488 \mathrm{~nm}$ line of an argon ion laser and fluorescence was measured at $515 \pm 15 \mathrm{~nm}$. Action potentials were induced by electrical field stimulation using a pair of platinum electrodes, which were connected to a Grass stimulator (Astro-Med. Inc., USA) set at a voltage $~ 50 \%$ above the threshold. Fluo-4 images were acquired in line-scan mode ( 3 ms per scan; pixel size 0.12 $\mu \mathrm{m})$. The scan line was positioned along a region of the cell where cell motion is minimal during contraction. Rabbit ventricular myocytes were studied in Tyrode solution containing (in $\mathrm{mM}$ ): $\mathrm{NaCl} 140 ; \mathrm{KCl} 4 ; \mathrm{CaCl}_{2} 2 ; \mathrm{MgCl}_{2}$ 1; glucose 10; HEPES 10; $\mathrm{pH}$ 7.4. Fish 
myocytes were studied in Tyrode solution containing (in $\mathrm{mM}$ ): $\mathrm{NaCl} 132 ; \mathrm{KCl} 2.5 ; \mathrm{MgCl}_{2}$ 1.6; $\mathrm{NaH}_{2} \mathrm{PO}_{4}$ 0.33; $\mathrm{NaHCO}_{3} 4 ; \mathrm{CaCl}_{2} 2$; glucose 5; Na pyruvate 5 and HEPES 10; $\mathrm{pH}$ 7.5.

Measurements of $\left[\mathrm{Ca}^{2+}\right]_{i}$ in permeabilized myocytes-Myocytes were permeabilized with saponin [32]. The saponin free internal solution was composed of (in $\mathrm{mM}$ ): potassium aspartate $100 ; \mathrm{KCl} 15 ; \mathrm{KH}_{2} \mathrm{PO}_{4} 5 ; \mathrm{MgATP}$ 5; EGTA 0.35; $\mathrm{CaCl}_{2}$ 0.12; $\mathrm{MgCl}_{2}$ 0.75; phosphocreatine 10; Hepes 10; Rhod-2 tripotassium salt 0.04; creatine phosphokinase $5 \mathrm{U} \mathrm{ml}^{-1}$; dextran (MW: 40,000) 8\%; pH 7.2 (KOH). Free $\left[\mathrm{Ca}^{2+}\right]$ and $\left[\mathrm{Ca}^{2+}\right]$ of this solution were $150 \mathrm{nM}$ and $1 \mathrm{mM}$, respectively. Rhod-2 was excited with the $543 \mathrm{~nm}$ line of a He-Ne laser and fluorescence was measured at wavelengths $>600 \mathrm{~nm}$. Images were acquired in line-scan mode ( $3 \mathrm{~ms}$ per scan; pixel size $0.12 \mu \mathrm{m}$ ). $\mathrm{Ca}^{2+}$ sparks were detected and analyzed using SparkMaster [23].

Labeling of the surface membrane-The density of the t-tubular system was measured after staining the surface membrane of rabbit and zebrafish myocytes with the voltagesensitive fluorescent dye Di-8-ANEPPS. Cells were incubated with extracellular solution containing $5 \mu \mathrm{M}$ of Di-8-ANEPPS for $15 \mathrm{~min}$. The Di-8-ANEPPS was excited at $488 \mathrm{~nm}$ and the fluorescence was recorded at $>600 \mathrm{~nm}$.

\section{Western blot analysis of RyR and RyR phosphorylation}

To prepare tissue samples, zebrafish hearts (from 6 fish) and pieces of rabbit ventricle (from 3 rabbits) were homogenized in saline buffer containing a protein inhibitors cocktail (Complete Mini, Roche, USA). Total protein concentration was quantified using Coomassie Plus reaction (Thermoscientific, USA). $20 \mu \mathrm{g}$ of each sample was subjected to 4-15\% SDSPAGE and transferred onto nitrocellulose membrane using Turbo-transfer (Bio-Rad, USA). The RyR expression level was assessed with the 34C primary antibody (DSHB, USA) and normalized to the total level of protein (Ponceau). RyR phosphorylation at the PKA site was measured in three different tissue samples (12 zebrafish hearts total): control, forskolin (5 $\mu \mathrm{M})$ and forskolin $(5 \mu \mathrm{M})$ plus the phosphatase inhibitor okadaic acid $(500 \mathrm{nM})$. The last sample was used to quantify the maximum level of RyR phosphorylation. Afterwards each sample was homogenized in Laemmli buffer and the same amount of total homogenate was subjected to 4-15\% SDS-PAGE and western blot. RyR phosphorylation at the PKA site (Ser 2715) was detected using a phospho-specific antibody RyR-Ser 2809 (Badrilla; UK). The signal was normalized to the total RyR level measured with the 34C antibody. The secondary antibodies were conjugated with horseradish peroxidase (HRP). The signals obtained with the HRP substrate (Luminata forte, Millipore, USA) were quantified using the UVP EpiChemi imaging system and ImageJ software.

\section{Statistics}

Data are presented as mean \pm SEM of $n$ measured cells. Statistical comparisons between groups were performed by the Student's $t$ test. Differences were considered statistically significant at $P<0.05$. 


\section{RESULTS}

Consistent with previous works [10;30], zebrafish ventricular myocytes had an elongated shape (width to length ratio is 0.09 ) compared to rabbit myocytes (ratio is 0.26 ).

Furthermore, staining of myocytes with Di-8-ANEPPS revealed that zebrafish ventricular myocytes lack t-tubular organization (Fig. 1).

\section{SR $\mathrm{Ca}^{2+}$ release during ECC in zebrafish ventricular myocytes}

In the following experiments, we compared the contribution of RyR and LTCC to the global $\mathrm{Ca}^{2+}$ transient during ECC in zebrafish and rabbit ventricular myocytes. Figs $2 \mathrm{~A}$ and B show line-scan images of $\left[\mathrm{Ca}^{2+}\right]_{\mathrm{i}}$ and corresponding profiles of $\mathrm{Ca}^{2+}$ transients from zebrafish and rabbit ventricular myocytes. $\mathrm{Ca}^{2+}$ transients were evoked by electrical field stimulation $(0.75 \mathrm{~Hz})$ to induce an AP or by caffeine $(10 \mathrm{mM})$ application to release $\mathrm{Ca}^{2+}$ stored in the SR. Despite a relatively similar $\mathrm{SR} \mathrm{Ca}^{2+}$ content in both species, the APinduced $\mathrm{Ca}^{2+}$ transient amplitude was smaller in zebrafish myocytes compared to rabbit myocytes. Since the SR is entirely depleted of $\mathrm{Ca}^{2+}$ in the presence of high doses of caffeine, the first AP-induced $\mathrm{Ca}^{2+}$ transient after caffeine application is entirely mediated by LTCC $\mathrm{Ca}^{2+}$ current. Indeed, a selective LTCC inhibitor verapamil $(10 \mu \mathrm{M})$ applied immediately after the caffeine application prevented any increase in $\left[\mathrm{Ca}^{2+}\right]_{\mathrm{i}}$ in myocytes from both species (data not shown). AP-induced $\mathrm{Ca}^{2+}$ transients measured in the presence of the SERCA inhibitor thapsigargin (TG; $5 \mu \mathrm{M}$ ) revealed similar amplitude compared to those recorded immediately following caffeine application (Fig 2, C). These results indicate that contribution of SR $\mathrm{Ca}^{2+}$ release to the first AP-induced $\mathrm{Ca}^{2+}$ transient following caffeine application is negligible. By analyzing $\mathrm{Ca}^{2+}$ transients before and after caffeine application, we found that in zebrafish myocytes $84.1 \pm 3 \%(\mathrm{n}=10)$ of the global $\mathrm{Ca}^{2+}$ transient during ECC was mediated by $\mathrm{Ca}^{2+}$ entry via LTCC and only $15.9 \pm 3 \%(\mathrm{n}=10)$ was due to $\mathrm{Ca}^{2+}$ release from the SR (Fig 3, A). In agreement with previous works [2;3], we found that in rabbit myocytes the contribution of LTCCs and RyRs to the AP-induced $\mathrm{Ca}^{2+}$ transient is $44 \pm 5 \%(\mathrm{n}=10)$ and $55 \pm 4 \%(\mathrm{n}=10)$, respectively (Fig 3, A). These data indicate that the contribution of LTCC and RyR to the amplitude of the $\mathrm{Ca}^{2+}$ transient during ECC is significantly different between zebrafish and rabbit myocytes.

Next, we analyzed the fractional SR $\mathrm{Ca}^{2+}$ release during the AP to estimate the sensitivity of RyR to the trigger (i.e. LTCC current) in zebrafish myocytes. In order to accurately determine the fractional release, the amplitude of the LTCC-mediated $\mathrm{Ca}^{2+}$ transient (measured immediately following caffeine application) was first subtracted from the amplitude of the AP-induced $\mathrm{Ca}^{2+}$ transient (measured before caffeine application). The fractional $\mathrm{SR} \mathrm{Ca}^{2+}$ release was then calculated as the ratio between the obtained value and the amplitude of the caffeine-induced $\mathrm{Ca}^{2+}$ transient. According to this analysis, in zebrafish myocytes only $\sim 9 \%$ of the $\mathrm{SR} \mathrm{Ca}^{2+}$ load is released into the cytosol during the $\mathrm{AP}$, whereas in rabbit this value is $\sim 40 \%$ (Fig 3, B). These results indicate that in zebrafish myocytes only a small fraction of $\mathrm{Ca}^{2+}$ stored in the SR contributes to ECC. This can be explained either by low sensitivity of the RyR to trigger $\mathrm{Ca}^{2+}$ or by a low expression level of RyRs in zebrafish heart. 


\section{RyR expression level in zebrafish ventricular myocytes}

In order to investigate the expression of RyRs in zebrafish and in rabbit hearts, western blot analysis with a monoclonal antibody against RyR (type 2) was performed. Fig 3, C shows that in zebrafish, this antibody could detect a band which corresponds to MW $\sim 560 \mathrm{kDa}$ with a signal density that is significantly lower (by $\sim 78 \%$ ) in comparison to rabbit hearts. However, it should be noted that this value could be overestimated due to a different immunoreactivity of the used antibody towards the zebrafish cardiac RyR.

\section{The sensitivity of CICR in zebrafish ventricular myocytes}

In the following experiments we studied $\mathrm{SR} \mathrm{Ca}^{2+}$ release in permeabilized zebrafish ventricular myocytes. The advantage of the membrane permeabilization is that an experimental solution with known $\left[\mathrm{Ca}^{2+}\right]$ can be easily introduced into the cytosol. We could not detect any $\mathrm{Ca}^{2+}$ spark activity at $100 \mathrm{nM}\left[\mathrm{Ca}^{2+}\right]_{\mathrm{i}}$. The increase of $\left[\mathrm{Ca}^{2+}\right]_{\mathrm{i}}$ up to $350 \mathrm{nM}$, a concentration that in permeabilized rabbit myocytes produces $\mathrm{Ca}^{2+}$ waves [9], also did not induce spark activity (data not shown). However, application of caffeine (10 $\mathrm{mM})$ at the end of each experiment produced a large $\mathrm{Ca}^{2+}$ transient, indicating that the absence of sparks was not due to depleted $\mathrm{SR} \mathrm{Ca}^{2+}$ load, but rather to low RyR activity or distribution of RyRs on the SR as isolated channels.

In order to increase the RyR activity, we used different concentrations of caffeine. The application of $200 \mu \mathrm{M}$ caffeine induced $\mathrm{Ca}^{2+}$ sparks at a frequency of 3.5 sparks $\times \mathrm{s}^{-1} \times(100$ $\mu \mathrm{m})^{-1}$ (Fig. 4, A). Caffeine (1 mM) increased RyR activity to the point that all the $\mathrm{SR} \mathrm{Ca}^{2+}$ release events were detected in the first $500 \mathrm{~ms}$ of the caffeine application (Fig 4, B). Further increase of the caffeine concentration to $5 \mathrm{mM}$ led to more synchronized $\mathrm{SR} \mathrm{Ca}^{2+}$ release: isolated $\mathrm{Ca}^{2+}$ sparks were no longer detectable because the simultaneous activation of RyRs produced a global $\mathrm{Ca}^{2+}$ transient (Fig 4, C). These results revealed that in zebrafish myocytes RyRs are organized in the form of release clusters, however, these release clusters exhibit a low sensitivity to $\left[\mathrm{Ca}^{2+}\right]_{\mathrm{i}}$.

\section{Effect of RyR phosphorylation by PKA on SR $\mathrm{Ca}^{2+}$ release during ECC}

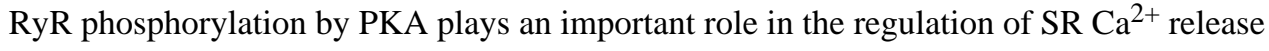
in the mammalian heart $[20 ; 21 ; 27]$. Thus, in the following experiments we investigated the susceptibility of the zebrafish RyR to PKA phosphorylation. First, we analyzed whether the zebrafish RyR amino acid sequence contained the consensus sites for PKA phosphorylation. Using "Blast P" software, we identified a PKA consensus site in the zebrafish RyR at Ser 2715 corresponding to the characterized Ser 2809 in the rabbit RyR. Using western blot analysis we measured the level of phosphorylation at Ser 2715. The basal level of phosphorylation at this site was $\sim 38 \%$. The treatment of zebrafish hearts with the adenylate cyclase activator forskolin $(5 \mu \mathrm{M})$ almost doubled the RyR phosphorylation level (Fig 5, A). These values were obtained after normalization to the maximal level of phosphorylation achieved in the presence of both forskolin and the phosphatase inhibitor okadaic acid.

We studied the effects of forskolin on $\mathrm{Ca}^{2+}$ release during ECC in intact zebrafish ventricular myocytes. In all myocytes studied, $\mathrm{Ca}^{2+}$ transient amplitude increased significantly upon application of forskolin $(5 \mu \mathrm{M})$. The increase was mainly due to 
augmentation of LTCC activity (as can be seen from the increased LTCC-mediated $\mathrm{Ca}^{2+}$ transients in Figs 5 B and C). However, the effect of forskolin on the RyR activity was more complex. In half of the studied cells, forskolin increased $\mathrm{SR} \mathrm{Ca}^{2+}$ load without a significant effect on RyR-mediated $\mathrm{Ca}^{2+}$ release (Fig 5, B). These results suggest that in these cells, PKA activation has a more profound effect on SERCA-mediated $\mathrm{Ca}^{2+}$ uptake than on RyRmediated $\mathrm{Ca}^{2+}$ release. Furthermore, these myocytes commonly responded to forskolin by producing a second peak during the $\mathrm{Ca}^{2+}$ transient indicating early after depolarization (EAD) (Fig 6, A). In the other half of the cells, the application of forskolin enhanced RyRmediated $\mathrm{Ca}^{2+}$ release and decreased SR Ca ${ }^{2+}$ load (Fig. 5, C). Thus, in these cells PKA activation had a more significant effect on RyR activity and diastolic $\mathrm{SR} \mathrm{Ca}^{2+}$ leak, than on SERCA activity. In these myocytes, the increased diastolic $\mathrm{Ca}^{2+}$ leak was commonly associated with $\mathrm{Ca}^{2+}$ spark activity (Fig 6, B). However, none of these cells showed $\mathrm{Ca}^{2+}$ waves or delayed after depolarization (DAD).

\section{Effect of RyR phosphorylation by PKA on spontaneous SR $\mathrm{Ca}^{2+}$ release}

We also studied the effect of PKA activation on $\mathrm{SR} \mathrm{Ca}^{2+}$ release in permeabilized zebrafish ventricular myocytes. As described above, zebrafish myocytes did not exhibit any detectable SR $\mathrm{Ca}^{2+}$ release events at high $\left[\mathrm{Ca}^{2+}\right]_{i}$. To maximally increase RyR phosphorylation at the PKA site, the internal solution containing cAMP $(20 \mu \mathrm{M})$, the PKA catalytic subunit (5 $\mathrm{U} / \mathrm{ml})$ and the phosphatase inhibitor okadaic acid $(100 \mathrm{nM})$ were introduced into the cytosol. The application of this "phosphorylation cocktail" caused a rapid rise in $\mathrm{Ca}^{2+}$ spark frequency in half of the studied cells. After reaching a peak within $1 \mathrm{~min}, \mathrm{Ca}^{2+}$ spark frequency began to gradually decline and after $3 \mathrm{~min} \mathrm{Ca}^{2+}$ sparks could not be detected (Fig 7 , B). Spontaneous $\mathrm{Ca}^{2+}$ release events recorded in zebrafish myocytes had an average amplitude of $0.35 \pm 0.07 \Delta \mathrm{F} / \mathrm{F}_{0}(\mathrm{n}=10)$.

\section{DISCUSSION}

Results in this study revealed that in zebrafish ventricular myocytes the cytosolic $\mathrm{Ca}^{2+}$ transient induced by the AP is mainly mediated by $\mathrm{Ca}^{2+}$ influx via LTCCs, whereas only a small fraction of $\mathrm{Ca}^{2+}(<20 \%)$ is released from the SR via RyRs. Such composition of $\mathrm{Ca}^{2+}$ transients is considerably different from the one reported in adult mammalian cardiomyocytes (e.g. rabbit, human) where $\mathrm{SR} \mathrm{Ca}^{2+}$ release contributes to nearly $70 \%$ of the $\mathrm{Ca}^{2+}$ transient during ECC (Fig 3 and also see [4]). Despite a small contribution of the SR to the $\mathrm{Ca}^{2+}$ transient, the $\mathrm{SR}$ of zebrafish myocytes is able to retain a significant amount of $\mathrm{Ca}^{2+}$ as demonstrated from the caffeine-induced $\mathrm{Ca}^{2+}$ release (Fig 2). Thus, the diminished $\mathrm{SR} \mathrm{Ca}^{2+}$ release during ECC in zebrafish myocytes is likely to be the result of lower activity of RyRs. The spatial separation of triggering LTCCs and RyRs due to the lack of the ttubular system (Fig 1) could reduce the efficacy of CICR contributing to the decreased SR $\mathrm{Ca}^{2+}$ release. It seems, however, that the lack of the t-tubular system in zebrafish ventricular myocytes is compensated by their small volume and elongated shape as in mammalian atrial cells [7]. Therefore, $\mathrm{Ca}^{2+}$ that enters the cytosol via LTCCs propagates throughout the cell without any detectable delay (Fig $2 ; \mathrm{Ca}^{2+}$ transients mediated by LTTCs). Therefore, other factors may play a role in the diminished $\mathrm{SR} \mathrm{Ca}^{2+}$ release in zebrafish myocytes, including low expression level of the RyR. Our data show that in the zebrafish heart the density of the 
band corresponding to the RyR is at least $78 \%$ lower than that found in rabbit myocardium. However, this difference might be an underestimation of the RyR expression level in zebrafish hearts due to the poor immunoreactivity of the antibody towards the fish cardiac RyR isoform.

The entire amino acid sequence of the zebrafish cardiac RyR (type 2) has not been completely determined. The characterized sequences of the RyR from zebrafish and rabbit share $\sim 85 \%$ similarity. Therefore, the unknown domains might be profoundly different from the rabbit RyR. Moreover, differences in the amino acid sequences between these two species might be located within some important regulatory sites of the channel. For instance, activation of the mammalian RyR is mediated by the high affinity cytosolic $\mathrm{Ca}^{2+}$ binding site [14] composed of the glutamates at position 3885 of each RyR monomer [11]. Because this region of the zebrafish RyR has not been determined, there might be crucial amino acid differences that decrease the channel sensitivity to $\left[\mathrm{Ca}^{2+}\right]_{\mathrm{i}}$. This would lead to a smaller SR $\mathrm{Ca}^{2+}$ release during the AP in zebrafish myocytes. It can also explain the absence of spontaneous $\mathrm{Ca}^{2+}$ sparks at $350 \mathrm{nM}\left[\mathrm{Ca}^{2+}\right]_{i}$, a concentration that triggers $\mathrm{Ca}^{2+}$ waves in rabbit myocytes [9]. Clearly our spark data suggest that RyRs in zebrafish myocytes are significantly less sensitive to $\left[\mathrm{Ca}^{2+}\right]_{\mathrm{i}}$ than the cardiac RyR in mammals. Low activity of the RyR has also been reported in trout ventricular myocytes [25]. It has been suggested that in trout myocytes the absence of $\mathrm{Ca}^{2+}$ sparks at high $\left[\mathrm{Ca}^{2+}\right]_{\mathrm{i}}$ is a result of the lack of continuity in the RyR cluster organization. In zebrafish myocytes, however, it was not the case, since the application of $200 \mu \mathrm{M}$ caffeine in permeabilized myocytes triggered $\mathrm{Ca}^{2+}$ sparks with spatio-temporal characteristics similar to a typical mammalian cardiac spark. It is well established that the propensity of spontaneous $\mathrm{Ca}^{2+}$ release from the SR directly depends on $\mathrm{SR} \mathrm{Ca}^{2+}$ load [18;24;31]. We found that the SR of zebrafish myocytes contains a significant amount of $\mathrm{Ca}^{2+}$ at rest (Fig 4). However, the role of this $\mathrm{SR} \mathrm{Ca}^{2+}$ content that is not fully used on a beat to beat basis remains elusive. The fact that the SR of zebrafish myocytes is equipped with $\mathrm{Ca}^{2+}$ release and uptake machineries suggests that this $\mathrm{Ca}^{2+}$ load could be used to increase heart contraction during stimulation of certain signaling pathways that sensitize the RyR to $\left[\mathrm{Ca}^{2+}\right]_{\mathrm{i}}$ (similar to the caffeine effect).

In mammalian myocytes, the RyR-mediated $\mathrm{Ca}^{2+}$ release can be significantly increased by $\beta$-adrenergic receptor ( $\beta$-AR) stimulation [4]. The stimulation of $\beta$-ARs leads to activation of the adenylate cyclase-PKA pathway with following phosphorylation of several important proteins involved in ECC, including the RyR [8;16;20;29]. Although controversial [6;26], it has been shown that phosphorylation of mammalian RyR at Ser 2808 by PKA increases RyR activity and $\mathrm{SR} \mathrm{Ca}^{2+}$ release [20;27;29]. In zebrafish ventricular myocytes, the role of PKA phosphorylation as a possible mechanism that can "unlock" the RyR remains obscure. We found that in zebrafish myocytes activation of adenylate cyclase by forskolin application increased phosphorylation of the RyR at the PKA site (Fig 5A). We also found that in intact myocytes forskolin increased the amplitude of AP-induced $\mathrm{Ca}^{2+}$ transients (Figs $5 \mathrm{~B}$ and $\mathrm{C}$ ). However, the contributions of LTCC and RyR to the global $\left[\mathrm{Ca}^{2+}\right]_{\mathrm{i}}$ increase varied significantly between cells. That is, in $\sim 50 \%$ of the myocytes the inotropic effect of forskolin was mainly mediated via augmentation of $\mathrm{Ca}^{2+}$ current via LTCCs, without any significant effect on $\mathrm{SR} \mathrm{Ca}^{2+}$ release. In contrast, in the other myocytes the forskolin inotropic action was associated with an increase of both LTCC-mediated $\mathrm{Ca}^{2+}$ current and 
RyR-mediated $\mathrm{Ca}^{2+}$ release. In rabbit myocytes increased RyR sensitivity during $\beta$-AR stimulation causes $\mathrm{SR} \mathrm{Ca}^{2+}$ leak in the form of sparks and waves [8]. In zebrafish myocytes that are characterized by increased RyR activity, the forskolin application also increased SR $\mathrm{Ca}^{2+}$ leak in the form of diastolic $\mathrm{Ca}^{2+}$ sparks (Fig 6). As a result, the increased $\mathrm{SR} \mathrm{Ca}^{2+}$ leak partially depleted SR $\mathrm{Ca}^{2+}$ load. In agreement with the intact cell results, we found that treatment of permeabilized myocytes with PKA triggered $\mathrm{Ca}^{2+}$ sparks only in half of the studied cells (Fig 7). We do not know the reason why only in some of zebrafish myocytes PKA activation increased $\mathrm{SR} \mathrm{Ca}^{2+}$ release. It is possible that high protein phosphatase activity in some cells prevents RyR phosphorylation from reaching a sufficient level that is required for the RyR activity.

The presented results highlight two important aspects of the zebrafish $\mathrm{SR} \mathrm{Ca}^{2+}$ handling that might be essential for considering this animal model to study human cardiac diseases associated with RyR mutations. The mechanism of ECC in zebrafish myocytes differs from that in mammalian myocardium mainly by the small contribution of SR $\mathrm{Ca}^{2+}$ release to the global $\mathrm{Ca}^{2+}$ transient. This difference could be a result of the zebrafish RyR structure that translates into a low sensitivity of the channel to $\left[\mathrm{Ca}^{2+}\right]_{\mathrm{i}}$. On the other hand, RyR phosphorylation by PKA appears to affect RyR function similarly in both mammalian and zebrafish myocytes. Further investigations of $\mathrm{SR} \mathrm{Ca}^{2+}$ release in zebrafish myocytes can provide important information how alterations of the RyR structure translate into altered function. Furthermore, the fact that RyRs in zebrafish myocytes are almost inactive under basal conditions provides an advantage for the generation of transgenic animals expressing human RyR mutations. In such transgenic models, the activity of the expressed RyR will be easy to study because it would not be contaminated by "noise" from endogenous zebrafish RyRs. Thus, the zebrafish may constitute an ideal model for investigation of human pathologies associated with RyR malfunction such as in heart failure.

\section{Acknowledgments}

We would like to thank Olga Alekhina for excellent technical assistance. This work was supported by the National Institutes of Health Grants HL62426 and HL75494 to P.d.T., The Research Career Development Award from The Schweppe Foundation to A.V.Z. and American Heart Association Fellowship Grant 14510047 to A.V.D.

\section{REFERENCE LIST}

1. Ai X, Curran JW, Shannon TR, Bers DM, Pogwizd SM. Ca2+/calmodulin-dependent protein kinase modulates cardiac ryanodine receptor phosphorylation and sarcoplasmic reticulum $\mathrm{Ca} 2+$ leak in heart failure. Circ Res. 2005; 97:1314-1322. [PubMed: 16269653]

2. Bassani JW, Bassani RA, Bers DM. Twitch-dependent SR Ca accumulation and release in rabbit ventricular myocytes. Am J Physiol. 1993; 265:C533-C540. [PubMed: 8368279]

3. Bassani JW, Bassani RA, Bers DM. Relaxation in rabbit and rat cardiac cells: species-dependent differences in cellular mechanisms. J Physiol. 1994; 476:279-293. [PubMed: 8046643]

4. Bers, DM. Excitation-Contraction Coupling and Cardiac Contractile Force. Dordrecht: Kluwer Academic Publishers; 2001.

5. Bers DM. Macromolecular complexes regulating cardiac ryanodine receptor function. J Mol Cell Cardiol. 2004; 37:417-429. [PubMed: 15276012]

6. Bers DM. Ryanodine receptor S2808 phosphorylation in heart failure: smoking gun or red herring. Circ Res. 2012; 110:796-799. [PubMed: 22427320] 
7. Blatter LA, Kockskamper J, Sheehan KA, Zima AV, Huser J, Lipsius SL. Local calcium gradients during excitation-contraction coupling and alternans in atrial myocytes. J Physiol. 2003; 546:19-31. [PubMed: 12509476]

8. Bovo E, Lipsius SL, Zima AV. Reactive oxygen species contribute to the development of arrhythmogenic $\mathrm{Ca} 2+$ waves during beta-adrenergic receptor stimulation in rabbit cardiomyocytes. J Physiol. 2012; 590:3291-3304. [PubMed: 22586224]

9. Bovo E, Mazurek SR, Blatter LA, Zima AV. Regulation of sarcoplasmic reticulum Ca2+ leak by cytosolic Ca2+ in rabbit ventricular myocytes. J Physiol. 2011; 589:6039-6050. [PubMed: 21986204]

10. Brette F, Luxan G, Cros C, Dixey H, Wilson C, Shiels HA. Characterization of isolated ventricular myocytes from adult zebrafish (Danio rerio). Biochem Biophys Res Commun. 2008; 374:143-146. [PubMed: 18602892]

11. Chen SR, Ebisawa K, Li X, Zhang L. Molecular identification of the ryanodine receptor Ca2+ sensor. J Biol Chem. 1998; 273:14675-14678. [PubMed: 9614063]

12. Domeier TL, Blatter LA, Zima AV. Alteration of sarcoplasmic reticulum Ca2+ release termination by ryanodine receptor sensitization and in heart failure. J Physiol. 2009; 587:5197-5209. [PubMed: 19736296]

13. Fabiato A. Calcium-induced release of calcium from the cardiac sarcoplasmic reticulum. Am J Physiol. 1983; 245:C1-14. [PubMed: 6346892]

14. Fabiato A. Time and calcium dependence of activation and inactivation of calcium-induced release of calcium from the sarcoplasmic reticulum of a skinned canine cardiac Purkinje cell. J Gen Physiol. 1985; 85:247-289. [PubMed: 2580043]

15. Fill M, Copello JA. Ryanodine receptor calcium release channels. Physiol Rev. 2002; 82:893-922. [PubMed: 12270947]

16. Huke S, Bers DM. Ryanodine receptor phosphorylation at Serine 2030, 2808 and 2814 in rat cardiomyocytes. Biochem Biophys Res Commun. 2008; 376:80-85. [PubMed: 18755143]

17. Lompre AM, Hajjar RJ, Harding SE, Kranias EG, Lohse MJ, Marks AR. Ca2+ cycling and new therapeutic approaches for heart failure. Circulation. 2010; 121:822-830. [PubMed: 20124124]

18. Lukyanenko V, Gyorke I, Gyorke S. Regulation of calcium release by calcium inside the sarcoplasmic reticulum in ventricular myocytes. Pflugers Arch. 1996; 432:1047-1054. [PubMed: 8781199]

19. Marks AR. Cardiac intracellular calcium release channels: role in heart failure. Circ Res. 2000; 87:8-11. [PubMed: 10884365]

20. Marx SO, Reiken S, Hisamatsu Y, Jayaraman T, Burkhoff D, Rosemblit N, Marks AR. PKA phosphorylation dissociates FKBP12.6 from the calcium release channel (ryanodine receptor): defective regulation in failing hearts. Cell. 2000; 101:365-376. [PubMed: 10830164]

21. Morimoto S, Uchi J, Kawai M, Hoshina T, Kusakari Y, Komukai K, Sasaki H, Hongo K, Kurihara S. Protein kinase A-dependent phosphorylation of ryanodine receptors increases $\mathrm{Ca} 2+$ leak in mouse heart. Biochem Biophys Res Commun. 2009; 390:87-92. [PubMed: 19781523]

22. Nemtsas P, Wettwer E, Christ T, Weidinger G, Ravens U. Adult zebrafish heart as a model for human heart? An electrophysiological study. J Mol Cell Cardiol. 2010; 48:161-171. [PubMed: 19747484]

23. Picht E, Zima AV, Blatter LA, Bers DM. SparkMaster: automated calcium spark analysis with ImageJ. Am J Physiol Cell Physiol. 2007; 293:C1073-C1081. [PubMed: 17376815]

24. Satoh H, Blatter LA, Bers DM. Effects of [Ca2+]i, SR Ca2+ load, and rest on $\mathrm{Ca} 2+$ spark frequency in ventricular myocytes. Am J Physiol. 1997; 272:H657-H668. [PubMed: 9124422]

25. Shiels HA, White E. Temporal and spatial properties of cellular $\mathrm{Ca} 2+$ flux in trout ventricular myocytes. Am J Physiol Regul Integr Comp Physiol. 2005; 288:R1756-R1766. [PubMed: 15650128]

26. Valdivia HH. Ryanodine receptor phosphorylation and heart failure: phasing out S2808 and “criminalizing” S2814. Circ Res. 2012; 110:1398-1402. [PubMed: 22628571]

27. Wehrens XH, Lehnart SE, Reiken S, Vest JA, Wronska A, Marks AR. Ryanodine receptor/calcium release channel PKA phosphorylation: a critical mediator of heart failure progression. Proc Natl Acad Sci U S A. 2006; 103:511-518. [PubMed: 16407108] 
28. Yano M. Ryanodine receptor as a new therapeutic target of heart failure and lethal arrhythmia. Circ J. 2008; 72:509-514. [PubMed: 18362417]

29. Yoshida A, Takahashi M, Imagawa T, Shigekawa M, Takisawa H, Nakamura T. Phosphorylation of ryanodine receptors in rat myocytes during beta-adrenergic stimulation. J Biochem. 1992; 111:186-190. [PubMed: 1349013]

30. Zhang PC, Llach A, Sheng XY, Hove-Madsen L, Tibbits GF. Calcium handling in zebrafish ventricular myocytes. Am J Physiol Regul Integr Comp Physiol. 2011; 300:R56-R66. [PubMed: 20926764]

31. Zima AV, Bovo E, Bers DM, Blatter LA. Ca2+ spark-dependent and -independent sarcoplasmic reticulum $\mathrm{Ca} 2+$ leak in normal and failing rabbit ventricular myocytes. J Physiol. 2010; 588:47434757. [PubMed: 20962003]

32. Zima AV, Picht E, Bers DM, Blatter LA. Partial inhibition of sarcoplasmic reticulum ca release evokes long-lasting ca release events in ventricular myocytes: role of luminal ca in termination of ca release. Biophys J. 2008; 94:1867-1879. [PubMed: 18024505] 


\section{Zebrafish}
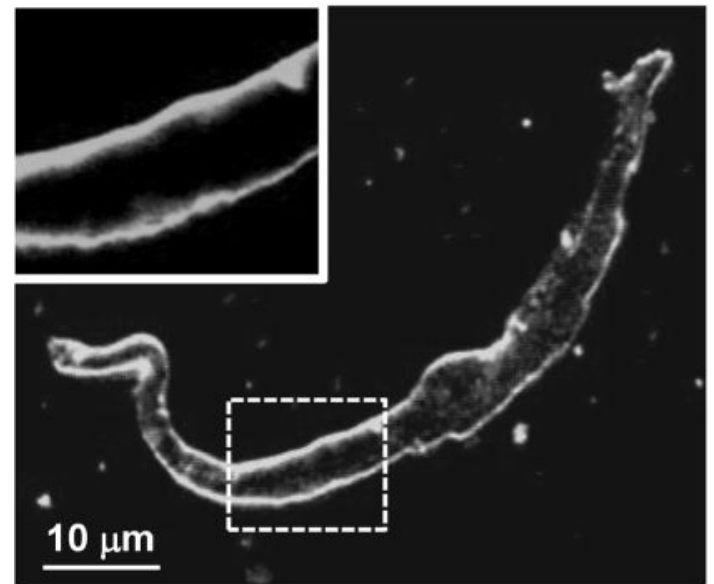

Rabbit

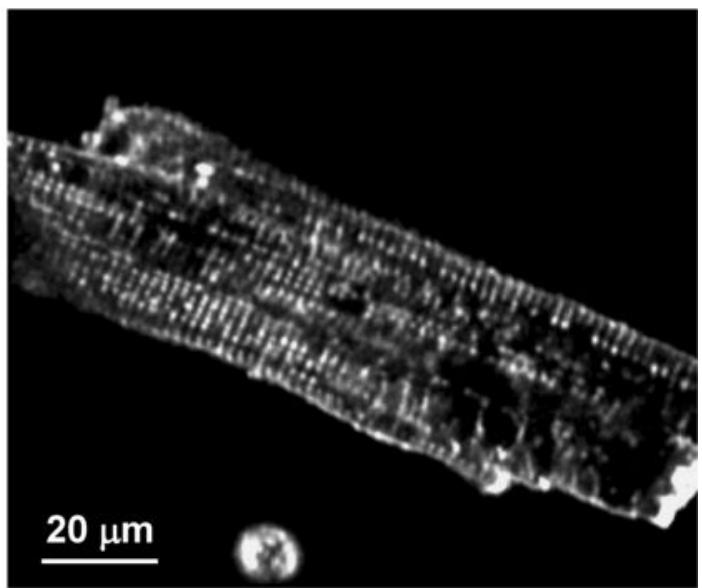

Figure 1. Morphology of zebrafish and rabbit ventricular myocytes Confocal 2-D images of ventricular myocytes from zebrafish (left panel) and rabbit (right panel) labeled with the voltage-sensitive fluorescent dye Di-8-ANEPPS. The left-top panel is an enlarged area marked with the dashed white line, to highlight the absence of the ttubular network in the zebrafish ventricular myocytes. 

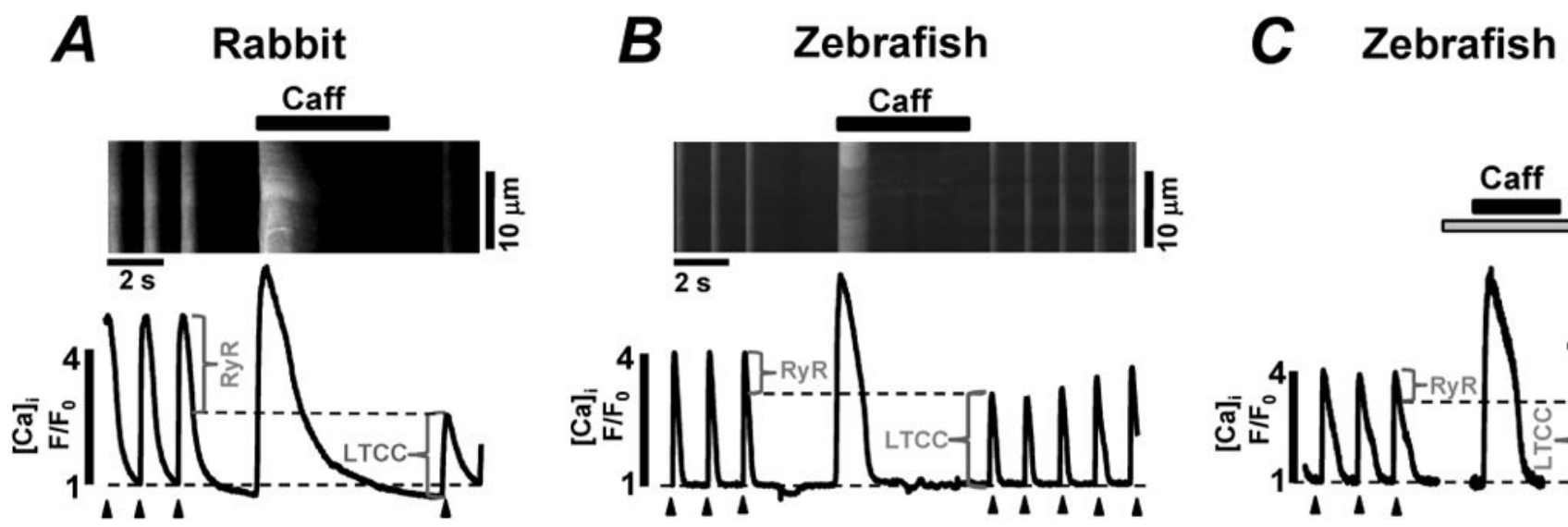

Figure 2. $\mathrm{Ca}^{2+}$ transients during ECC in zebrafish and rabbit ventricular myocytes Confocal line-scan images of Fluo-4 fluorescence and corresponding $\mathrm{F} / \mathrm{F}_{0}$ profiles recorded in rabbit $(\boldsymbol{A})$ and zebrafish $(\boldsymbol{B})$ ventricular myocytes. The $\mathrm{F} / \mathrm{F}_{0}$ profiles were obtained by averaging the fluorescence throughout the entire image. The myocytes were electrically stimulated at constant rate $(0.75 \mathrm{~Hz}$; marked by black arrowheads). Contributions of LTCC and RyR to the AP-induced $\mathrm{Ca}^{2+}$ transients are indicated by the brackets. $\boldsymbol{C}$, caffeineinduced and the AP-induced $\mathrm{Ca}^{2+}$ transients recorded in zebrafish ventricular myocytes in control conditions and after inhibition of SERCA with thapsigargin (TG; $10 \mu \mathrm{M})$. 

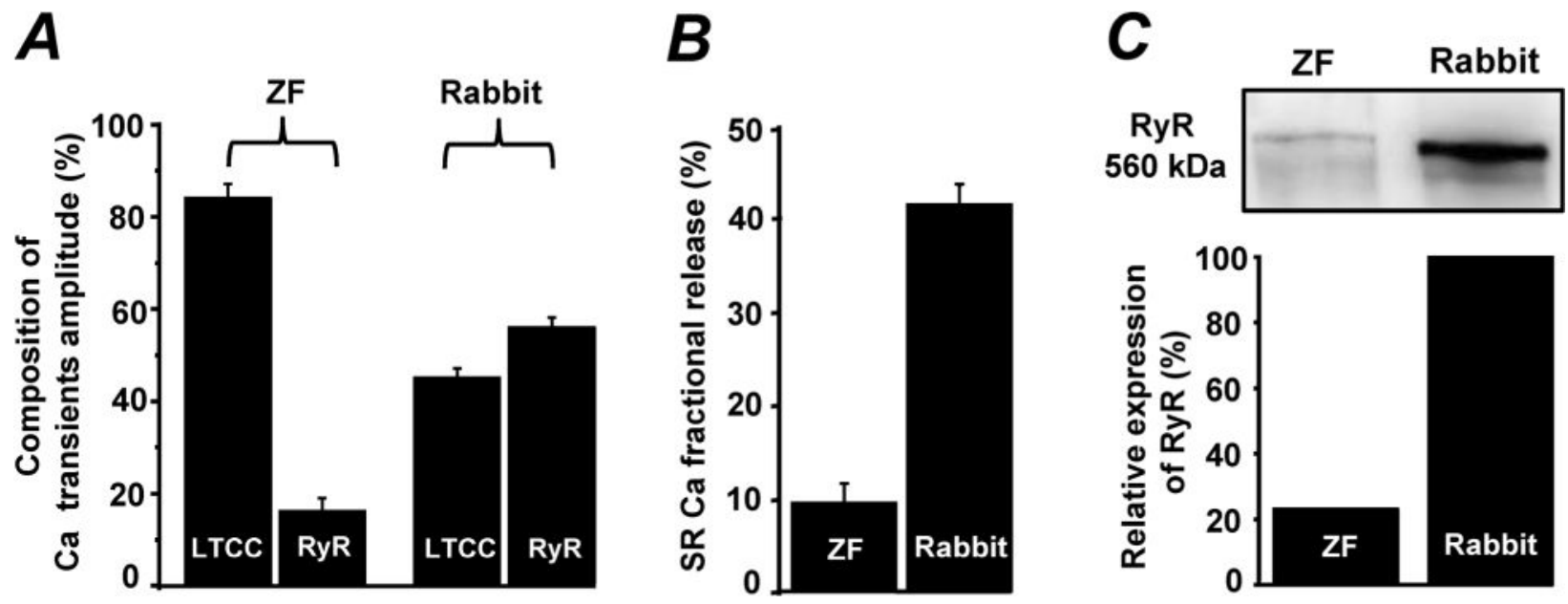

Figure 3. Contribution of LTCC and RyR to $\mathrm{Ca}^{2+}$ transients during ECC in zebrafish and rabbit ventricular myocytes

$\boldsymbol{A}$, components of the AP-induced $\mathrm{Ca}^{2+}$ transient in zebrafish (left part) and in rabbit (right part) ventricular myocytes. $\boldsymbol{B}$, fractional SR Ca release in zebrafish and rabbit ventricular myocytes. $\boldsymbol{C}$, representative western blots (top) and correspondent analysis (bottom) of the RyR expression level in zebrafish and rabbit hearts. The signal corresponding to the RyR level in zebrafish was normalized to the signal obtained in rabbit hearts. 

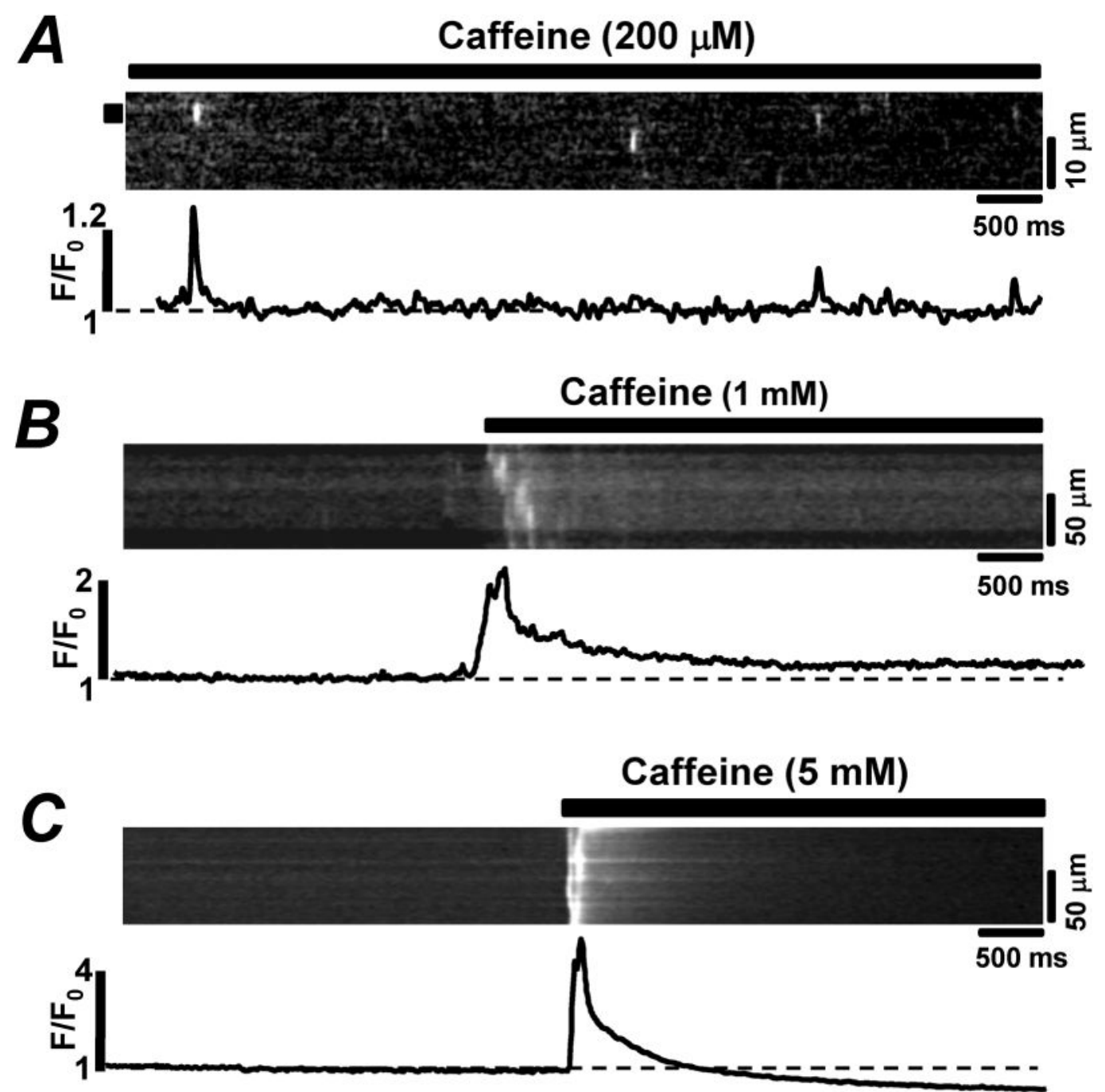

Figure 4. Effect of caffeine on RyR-mediated $\mathrm{Ca}^{2+}$ release in zebrafish ventricular myocytes Confocal line-scan images of Rhod- 2 fluorescence and corresponding $F / F_{0}$ profiles recorded in permeabilized zebrafish ventricular myocytes in the presence of $200 \mu \mathrm{M}(\boldsymbol{A}), 1 \mathrm{mM}(\boldsymbol{B})$ and $5 \mathrm{mM}(\boldsymbol{C})$ caffeine. Profiles were obtained by averaging the fluorescence throughout the area labeled by the black box $(\boldsymbol{A})$ or throughout the entire image ( $\boldsymbol{B}$ and $\boldsymbol{C}$ ). 

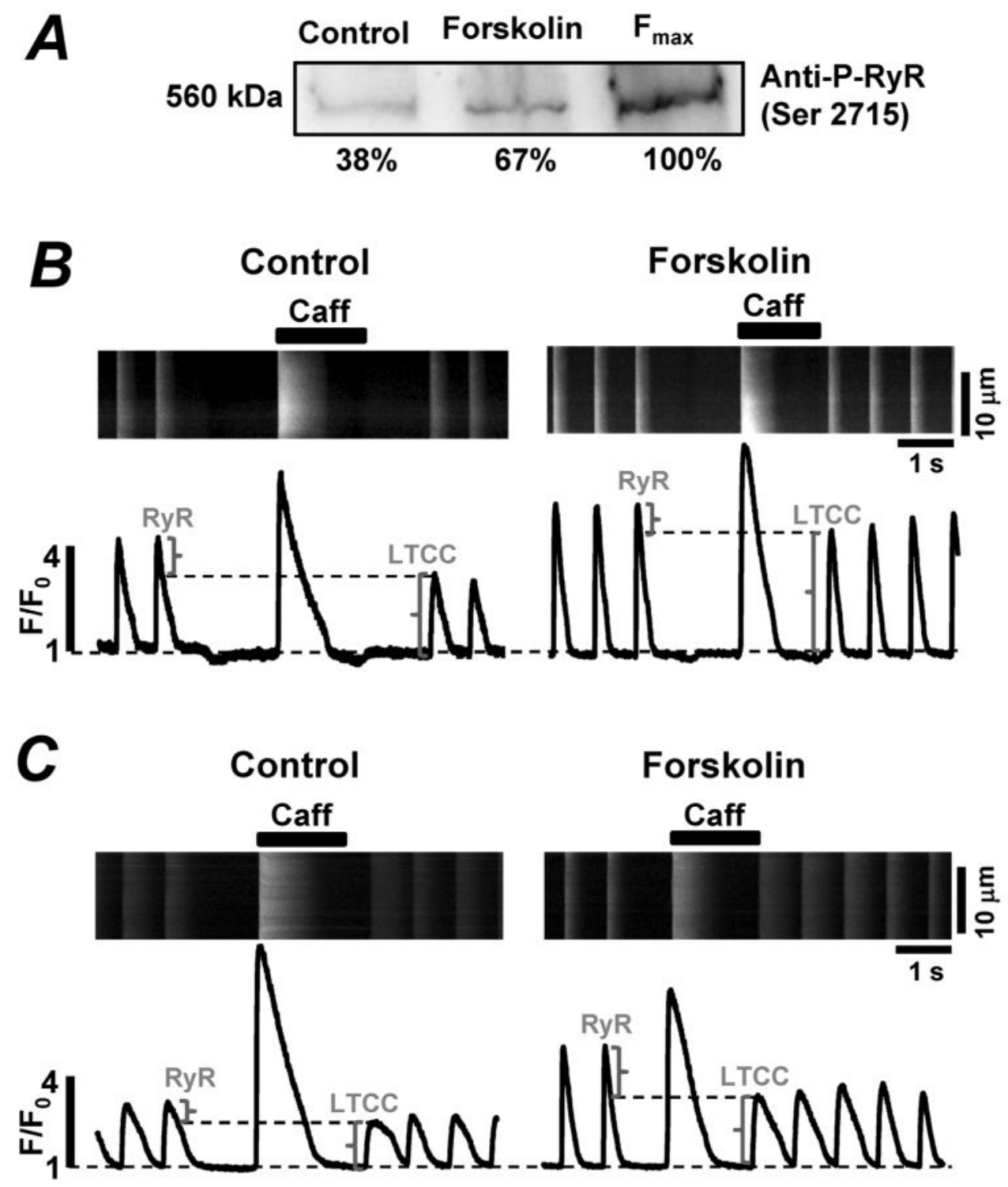

Figure 5. Effects of forskolin on RyR phosphorylation and $\mathrm{Ca}^{2+}$ transients in zebrafish ventricular myocytes

$\boldsymbol{A}$, representative western blots with corresponding statistical analysis showing the effect of forskolin $(5 \mu \mathrm{M})$ on RyR phosphorylation at the PKA specific site. The levels of phosphorylation were normalized to the maximal level of RyR phosphorylation at the PKA site (achieved after treatment of zebrafish hearts with forskolin and the phosphatase inhibitor okadaic acid). $\boldsymbol{B}, \boldsymbol{C}$, confocal line-scan images of Fluo-4 fluorescence and corresponding $\mathrm{F} / \mathrm{F}_{0}$ profiles recorded in zebrafish ventricular myocytes in control conditions (left panels) and in the presence of forskolin $(5 \mu \mathrm{M}$; right panels). The myocytes were electrically stimulated at $0.75 \mathrm{~Hz}$. The brackets indicate the contribution of RyR and LTCC to the APinduced $\mathrm{Ca}^{2+}$ transient. 

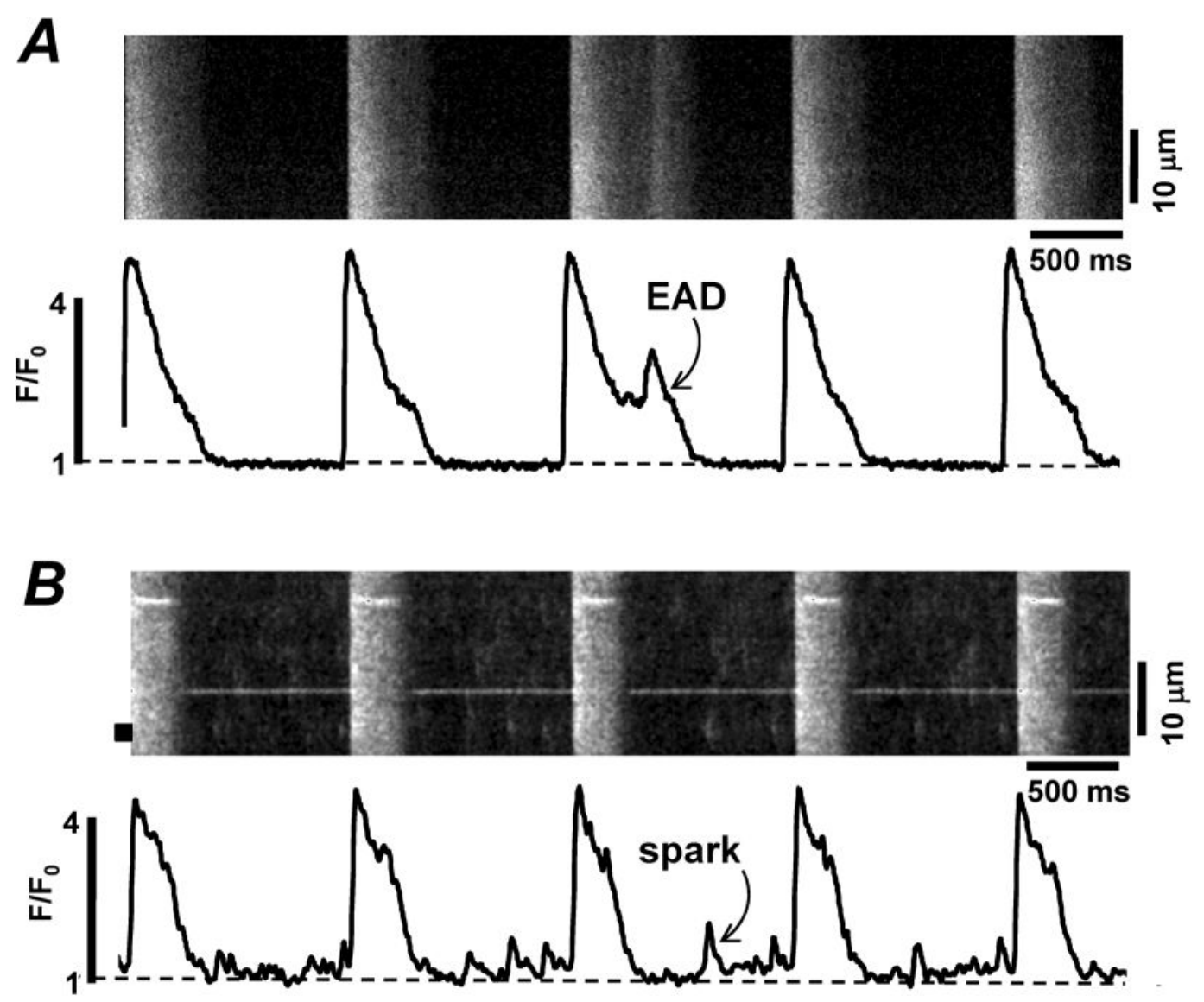

Figure 6. Effect of forskolin on $\mathrm{Ca}^{2+}$ transients in zebrafish ventricular myocytes Confocal line-scan images of Fluo-4 fluorescence and corresponding $\mathrm{F} / \mathrm{F}_{0}$ profiles recorded in electrically stimulated zebrafish myocytes during the application of forskolin $(5 \mu \mathrm{M}) . \boldsymbol{A}$, forskolin induced a second peak during the $\mathrm{Ca}^{2+}$ transient indicating early after depolarization (EAD). $\boldsymbol{B}$, forskolin produced diastolic $\mathrm{Ca}^{2+}$ sparks. 


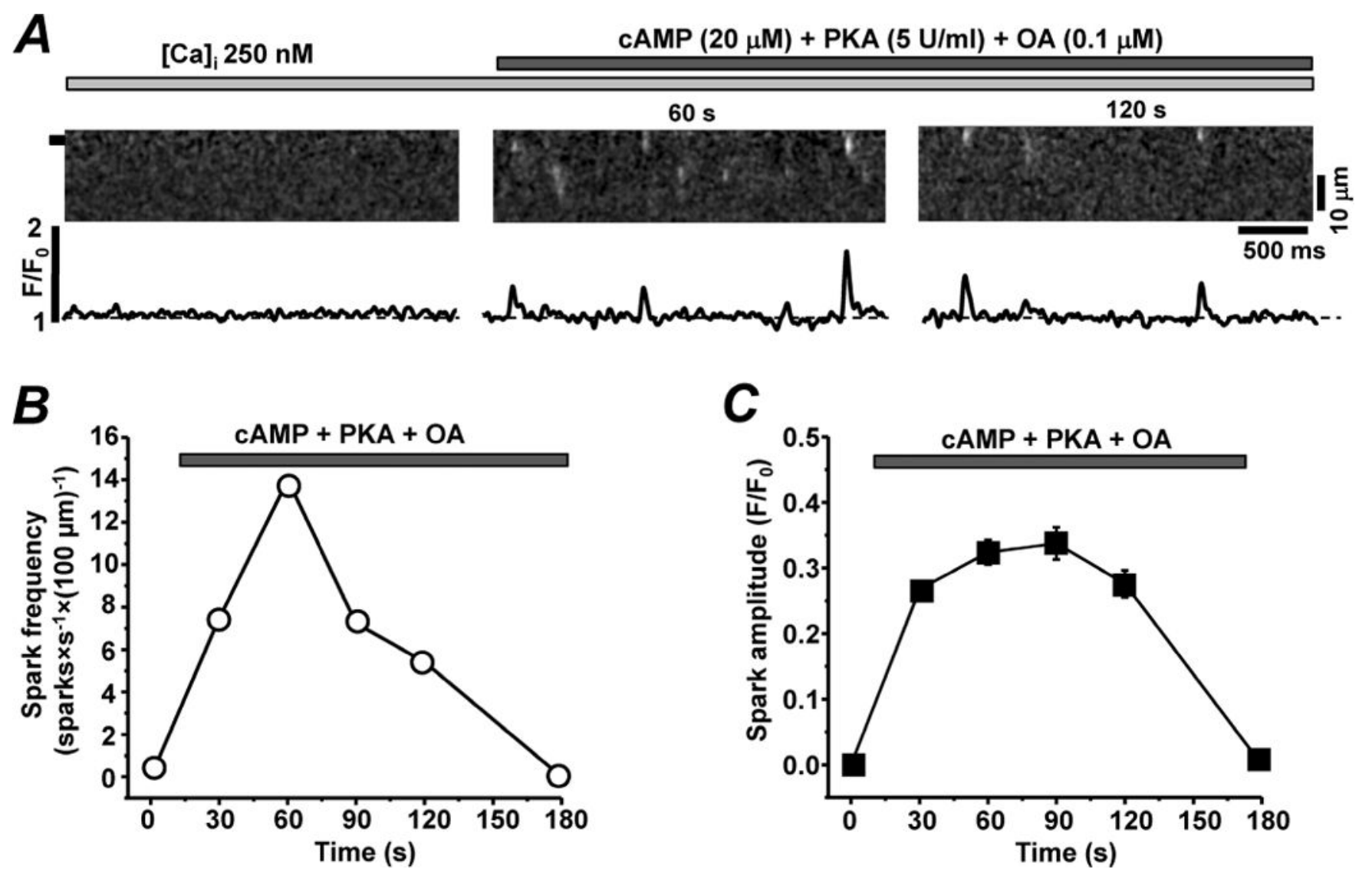

Figure 7. Effect of PKA activation on RyR-mediated $\mathrm{Ca}^{2+}$ release in zebrafish myocytes $\boldsymbol{A}$, confocal line-scan images of Rhod-2 fluorescence and corresponding $\mathrm{F} / \mathrm{F}_{0}$ profiles recorded in permeabilized zebrafish ventricular myocytes in the presence of $250 \mathrm{nM}\left[\mathrm{Ca}^{2+}\right]_{\mathrm{i}}$ (left panel) and after a subsequent application of cAMP $(20 \mu \mathrm{M})$, the PKA catalytic subunit $(5 \mathrm{U} / \mathrm{ml})$ and the phosphatase inhibitor okadaic acid $(\mathrm{OA} ; 100 \mathrm{nM}$; central and right panel). Profiles were obtained by averaging the fluorescence throughout the area labeled by the black box. Effects of PKA activation on Ca spark frequency $(\boldsymbol{B})$ and spark amplitude $(\boldsymbol{C})$. 